\title{
Formes et savoirs de la corruption à l'âge des Lumières
}

\section{Ronan Chalmin}

\section{(2) OpenEdition \\ 12 Journals}

Édition électronique

URL : http://journals.openedition.org/aes/1808

DOI : 10.4000/aes. 1808

ISSN : 2258-093X

Éditeur

Laboratoire LISAA

Référence électronique

Ronan Chalmin, «Formes et savoirs de la corruption à l'âge des Lumières », Arts et Savoirs [En ligne], 11 | 2019, mis en ligne le 15 juillet 2019, consulté le 05 septembre 2019. URL : http:// journals.openedition.org/aes/1808; DOI : 10.4000/aes.1808

Ce document a été généré automatiquement le 5 septembre 2019

Centre de recherche LISAA (Littératures SAvoirs et Arts) 


\title{
Formes et savoirs de la corruption à l'âge des Lumières
}

\author{
Ronan Chalmin
}

Plus l'intérieur se corrompt et plus l'extérieur se compose.

J.-J. Rousseau, Réponse à M. Bordes. ${ }^{1}$

1 Les philosophes et romanciers des Lumières ont fait de la réflexion sur la corruption le point de départ d'une mise en accusation des dysfonctionnements de la société d'Ancien Régime. Cette réflexion a également servi d'impulsion à une volonté de réformes qui devait conduire au grand bouleversement révolutionnaire ${ }^{2}$. Elle ne se cantonne pas, toutefois, au seul domaine de l'analyse philosophique ou politique. Les savants se penchent eux aussi sur le phénomène corrupteur dont ils tentent de mettre au jour les origines, les mécanismes et les conséquences. Par ailleurs, la critique de la corruption morale de l'individu vivant en société s'articule à une interrogation angoissée sur les effets nocifs de cette vie en société sur le corps humain. Car le corps - ce qui le constitue comme ce qui l'environne - est devenu un point de focalisation majeur pour les pourfendeurs de la corruption, tant sur le plan moral que médical. Ainsi, le Dr. Mauriac note au siècle suivant, dans un article consacré à l'onanisme, que « les débordements du XVIII ${ }^{\mathrm{e}}$ siècle donnèrent naissance à une littérature médicale et scientifique destinée à les combattre et accessible à tous les lecteurs qu'elle avait la prétention de moraliser " ${ }^{3}$. Quittant les arcanes du pouvoir ou les plis les plus enfouis du cœur et de l'esprit, la corruption s'exhibe au grand jour et se voit investie d'une forte polysémie : elle se lit sur les visages vérolés, s'observe dans les physiques dégénérés ou se respire dans les espaces peuplés et confinés. Le sang infecté par les virus et les venins charrie la maladie dans l'organisme humain comme dans l'organisme social. Un corps, puis un autre, puis un autre encore: tout n'est que circulation, contagion, contamination, pour sombrer en définitive dans une dégénérescence que seule la régénération physique et politique de la Révolution semble en mesure d'endiguer ${ }^{4}$. "Le pathos de la corruption marque spécialement certains esprits qui lui trouvent des origines mythiques ", explique Jacques Guillerme : «l'incessante production du putride indiquerait la fatale dégénérescence d’un 
état de pureté primordial $»^{5}$. Cette quête d'une pureté disparue alimente la pensée des Lumières.

Parallèlement à cette nostalgie de la pureté perdue, les savoirs de la corruption se multiplient et touchent à nombre de domaines scientifiques en pleine mutation. En tant qu' « état par lequel une chose cesse d'être ce qu'elle était $»^{6}$ - selon la définition qu'en donne l'Encyclopédie de Diderot et d'Alembert - elle suscite des travaux visant à une meilleure compréhension du phénomène complexe de la vie, que cette vie soit sociale ou animale ${ }^{7}$. Ainsi, paradoxalement, la corruption encourage le progrès: de nouvelles techniques et de nouvelles pratiques se développent pour mieux en contrecarrer les effets. De même, dans les salons ou les académies, dans les mémoires ou dans les encyclopédies, la corruption, la décomposition, la fermentation ou la putréfaction, à savoir tous ces phénomènes limites « au seuil de l'organique et du matériel, de la vie et de la mort $»^{8}$ sont au cœur des discussions. Face à la menace d'une dégénérescence irréversible, l'époque est acquise à la philosophie du progrès et à la volonté de perfectionner les constitutions et les institutions ${ }^{9}$ en inventant de nouvelles lois et en imaginant de nouveaux remèdes, qu'il s'agisse de l'aseptisation, de l'aération ou de l'inoculation. En cet âge des possibles, le nouveau mot d'ordre est «de faire vivre, d'entretenir et de maximiser la vie de la population $»^{10}$.

3 Aussi nous proposons-nous ici de reconsidérer le siècle des Lumières à travers son rapport à la corruption et à ses différentes expressions. Nous nous intéresserons en particulier à la manière dont les savoirs de la corruption identifient celle-ci comme menace tout en reconnaissant à la fois sa place dans le cycle vital et son rôle comme instrument de progrès, notamment avec la pratique de l'inoculation. Après avoir exploré les analyses de Stahl sur la corruptibilité du corps humain, nous élargirons ensuite notre analyse aux nouvelles méthodes utilisées contre la corruption de l'air (Tournon, Mercier) et celle du sang (Voltaire, Tronchin, Condamine). En conclusion, nous examinerons le lien profond qui unit corruption et génération dans le phénomène de la vie à l'appui des thèses de Diderot et de d'Alembert.

\section{De la corruption du corps humain}

" Génie vaste, pénétrant, précis, enrichi par les connaissances de toute espèce »" , GeorgErnst Stahl (1660-1734), professeur à l'université de Halle et médecin du roi de Prusse, demeure dans l'histoire des sciences le père fondateur de deux théories: celle du vitalisme, dans sa version animiste, et celle du phlogistique, ou principe du feu, dont il forge lui-même le mot $^{12}$. Ces deux théories, centrales dans le discours scientifique de l'époque, sont taxées aujourd'hui d'obscurantisme. Elles ont joué cependant un rôle fondamental dans l'évolution des sciences, notamment dans l'émergence de la chimie moderne incarnée par Lavoisier.

5 La réflexion scientifique de Stahl part d'un constat simple : le peu de connaissance que nous avons de la vie en soi : «J'eus beau chercher en effet », observe-t-il, « ce fut en vain ; car aucun des propagateurs de ces prétendues doctrines n'a jamais dit et démontré ce que c'est, en quoi consiste, d'où provient, par quels modes, par quels moyens se maintient et subsiste ce que nous appelons la vie; par quoi, enfin, et sous quel point de vue le corps est dit vivant ${ }^{13}$. La réponse est simple pour Stahl : c'est la corruptibilité même du corps humain qui explique son fonctionnement vital. C'est la corruption incessante de 
l'organisme qui prouve la vie. Cette corruption, précise Stahl, ne se trouve nulle part ailleurs; elle est «l'apanage des corps vivants $»^{14}$. Comme André Pichot le remarque, «Stahl n'est certes pas le premier à avoir remarqué que le corps des êtres vivants est éminemment corruptible, mais il est incontestablement le premier à avoir placé cette corruptibilité au centre de sa biologie »" Toutefois, ce qui frappe le plus Stahl, c'est « que ce corps matériel, si disposé d'ailleurs à la corruption, malgré cette étonnante aptitude, ne subisse jamais cet effet d'imminente corruptibilité, qu'il résiste à une si profonde disposition à la pourriture, et que, sans en être atteint ni souillé, il subsiste enfin et dure ainsi pendant une longue série d'années $»^{16}$. Stahl insiste ainsi sur la puissance conservatrice du corps, capable de résister contre sa décomposition, synonyme de disparition. La fragilité du corps humain est due à sa mixtion, au fait qu'il n'est pas constitué d'une seule et même matière : «Le corps humain, à cause de sa mixtion, a une propension très grande à la corruption, sa structure [...] est profondément exposée au dépérissement absolu ${ }^{17}$. Mixtion et corruption sont deux opérations corrélées et contraires, comme Gabriel François Venel le précise dans son long article « Chymie » de l'Encyclopédie:

Les deux effets généraux, primitifs, et immédiats de toutes les opérations chimiques, savoir la séparation et l'union des principes, sont plus connus sous le nom de diacrèse et syncrèse. La première est appelée aussi par plusieurs chimistes analyse, décomposition, corruption, solution, destruction; et la seconde, mixtion, génération, synthèse, combinaison, coagulation, et même confusion par certains [...]. ${ }^{18}$

6 La dichotomie dans la terminologie employée est tout à fait indicative de l'opération qui s'effectue au niveau chimique. Stahl oppose à la propension naturelle à la corruption un principe de conservation au fondement des mécanismes de la vie: "Il est donc évidemment nécessaire qu'il y ait en nous une force conservatrice; sa présence est même indispensable pour empêcher que la mixtion corporelle de l'agrégat ne tombe actuellement en corruption». Cette force conservatrice, suppose-t-il, doit être "durable et permanente dans ce même corps $»^{19}$. C'est ainsi que Stahl aboutit à une nouvelle définition de la vie comme résistance à la corruption du corps. Cette définition annonce la formulation de Xavier Bichat (1771-1802), qui déclarera dans son préambule aux Recherches physiologiques sur la vie et la mort (1800) que " la vie est l'ensemble des fonctions qui résistent à la mort » ${ }^{20}$. "Nous dirons donc», conclut Stahl, "que dans le langage ordinaire, on doit proprement entendre par ce mot, Vie: la conservation même d'un corps éminemment corruptible, la faculté ou force à l'aide de laquelle ce corps est mis à l'abri de l'acte corrupteur $\nu^{21}$. L'organisme est donc le siège d'un combat incessant visant à empêcher sa décomposition.

Une fois ce principe énoncé, Stahl se livre à une explication physiologique de la corruption du corps humain. Elle tient, selon lui, à la composition mucido-adipeuse de l'organisme. Si cette composition d'eau et de graisse assure la flexibilité du corps, elle en rend - par sa nature mixte - la cohésion instable. Seule l'âme est en mesure de préserver l'intégrité de l'organisme. Stahl s'appuie ici sur la tradition médicale hippocratique de la Natura medicatrix, selon laquelle le malade possède en lui-même les moyens de sa guérison 22. Sans cette autorégulation, l'organisme ne pourrait survivre. Le corps, pour Stahl, possède donc son propre principe réparateur, en la présence des organes soumis à l'action de l'âme ${ }^{23}$. Nulle aide externe, nul médecin, mais un usage interne du remède, puisé en soi :

C'est donc, je le répète, à l'aide de cette méthode que le corps vit et qu'il est conservé dans la pureté et l'intégrité de sa mixtion. [...] Telle est la médecine de la nature, cette 
force, cette puissance qu'Hippocrate avait déjà signalée comme pouvant guérir un grand nombre d'individus de leur affection sans le recours même de l'art médical. ${ }^{24}$ Stahl. Elle peut également résulter de facteurs externes. À ce titre, le savoir sur l'air connait une réorientation majeure au $\mathrm{XVIII}^{\mathrm{e}}$ siècle. En effet, sous l'impulsion du courant néo-hippocratique, "l'air n'est plus tant étudié comme lieu de la génération ou de l'épanouissement de la vitalité que comme le laboratoire de la décomposition ${ }^{28}$. On observe ainsi une hantise de l'air, plus particulièrement de l'air méphitique, tenu pour un symptôme et un agent propagateur de corruption : «Partout où se tenaient des réunions nombreuses, que ce fût dans un édifice isolé ou même dans une cité entière ", signale Richard Etlin, «la corruption de l'air surgit au XVIII ${ }^{\mathrm{e}}$ siècle comme un problème irritant " ${ }^{29}$. Ce problème de première importance appelle de nouvelles méthodes d'épuration. nouvelles conceptions de l'air en font un véhicule d'odeurs nauséabondes, de miasmes et de virus. Comme le rappelle Alain Corbin, «fétidité et humidité définissent la corruption $»^{31}$. En effet, la matière organique produit, en se désagrégeant, un air vicié et corrupteur, chargé de miasmes putrides et source de maladie ${ }^{32}$. Selon l'article «Putréfaction, Putréfier [Chimie] » de l'Encyclopédie, « la putréfaction est le dernier degré 
de la fermentation, on la regarde presque généralement comme l'extrême dissolution des corps qui se corrompent. Stahl veut que ce soit le dernier état de division où les mixtes conservent leur combinaison, et approchent le plus d'être des individus ${ }^{33}$. On voit ici tout le danger que peut représenter pour la médecine des Lumières la pestilence de l'organisme mort en pleine putréfaction, libérant dans l'air quantité d'éléments nocifs. De même, la respiration, symbole de vie, produit elle aussi une dangereuse corruption: "L'haleine est bien devenue un danger : la respiration et son "méphitisme" particulier sont perçus comme décomposition; le souffle est perçu comme excrément ${ }^{34}$. On assiste alors au développement de ce qu'on nomme une pensée miasmatique, fondée sur «la conviction selon laquelle la maladie se répand typiquement non par le contact personnel, mais par des émanations dégagées par l'environnement ${ }^{35}$.

Cette peur de l'air vicié a un arrière-plan médical et moral lié à la crainte de l'urbanisation. Si les charognes, les déchets et les excréments jonchent les espaces ruraux et urbains, leur présence est particulièrement envahissante dans la ville : « Sa corruption provient du sol qui lui communique ses exhalaisons délétères, son méphitisme, ses miasmes $\aleph^{36}$. Alexandre Tournon, de l'Académie royale d'Arras, dresse un panorama alarmiste de ces grandes villes, « où le soleil et l'air pénètrent avec peine, où le sol fétide surcharge l'atmosphère de miasmes dangereux, où les matières corrompues s'accumulent sans cesse, où des milliers d'habitations ne cessent de méphitiser l'air [...] $\aleph^{37}$. Louis Sébastien Mercier signale l'apparition d'un " mot nouveau ", " méphitisme », qui signifie "vapeur empoisonnée " ${ }^{38}$, et consacre tout un chapitre à l'air vicié dans son vaste Tableau de Paris publié entre 1782 et 1788 : Chacun, remarque-t-il, «a dans sa maison des magasins de corruption; il s'exhale une vapeur infecte de cette multitude de fosses d'aisance ${ }^{39}$. Par ailleurs, les bâtisses sur les ponts de la capitale sont non seulement laides, mais elles empêchent "d'emporter avec les vapeurs de la Seine tout l'air corrompu des rues qui aboutissent aux quais $»^{40}$, au point que «l'air hépatique qui s'exhalait de ces foyers de corruption, corrompait la viande, attaquait les matières d'or et d'argent $»^{41}$. De plus, les rues étroites, les égouts à ciel ouvert, les cimetières au cœur de la cité font que "l'atmosphère se corrompt, se charge de particules impures, et que cet air renfermé devient pesant et d'une influence maligne ${ }^{42}$. La littérature de l'époque abonde de descriptions d'un environnement malsain entraînant une mortalité accrue dans les zones à forte densité humaine.

Dans l'espace urbain, l'hôpital et la prison sont en ligne de mire. Ainsi, la prison reste au $\mathrm{XVIII} \mathrm{e}^{\mathrm{e}}$ siècle « le pire des scandales olfactifs » ${ }^{43}$. Bicêtre est «le réceptacle de tout ce que la société a de plus immonde et de plus vil », affirme Mercier : «Jusqu'à l'air du lieu que l'on sent ici jusqu'à 400 toises, tout vous dit que vous approchez d'un lieu de force, d'un asile de dégradation et d'infortune $\aleph^{44}$. Le discours médical rejoint ici le discours moral pour stigmatiser l'espace carcéral :

Tout d'abord le mal entre en fermentation dans les espaces clos de l'internement. Il a toutes les vertus qu'on prête à l'acide dans la chimie du xvIII ${ }^{e}$ siècle : ses fines particules, coupantes comme des aiguilles, pénètrent les corps et les cœurs aussi facilement que s'ils étaient des particules alcalines, passives et friables. ${ }^{45}$

L'air est vicié par la décomposition des corps «à l'air libre » ou par la fermentation qui s'opère dans les lieux d'internement. Dans son Bicêtre réformé, Musquinet de la Pagne constate que "ces salles ne représentent qu'un lieu affreux où tous les crimes réunis fermentent, et répandent pour ainsi dire autour d'eux, par la fermentation, une atmosphère contagieuse que respirent et qui semblent s'attacher à ceux qui l'habitent $[. .$. 
] $\aleph^{46}$. La prison enferme l'individu moralement corrompu, lequel produit du fait de son enfermement une matière elle aussi corrompue qui se propage par voie aérienne. Ainsi, paradoxalement, la prison est une machine à produire de la corruption autant qu'à la détruire. Foucault ne manqua pas de relever cette confusion de fonctions :

On voit naître, et se ramifier en tous sens les thèmes d'un mal, physique et moral tout ensemble, et qui enveloppe, dans cette indécision, des pouvoirs confus de corrosion et d'horreur. Règne alors une sorte d'image indifférenciée de la «pourriture» qui concerne aussi bien la corruption des mœurs que la décomposition de la chair [...]. ${ }^{47}$

L'entassement des prisonniers forme le bouillon de culture d'un mal aussi bien moral que physique. Si la corruption morale de l'homme lors de sa socialisation a été mise en évidence par Jean-Jacques Rousseau ${ }^{48}$, la science médicale et hygiénique continue d'insister sur la corruption désormais physique de la concentration humaine dans les grandes agglomérations. Dans son rapport de 1788 sur la voirie de Montfaucon, le médecin Michel-Augustin Thouret (1748-1810) tire la sonnette d'alarme : «Il faut avoir parcouru ces lieux d'infection pour savoir ce que sont ces résidus ou produits, que l'on peut appeler les excréments d'une grande ville, et pour connaître quelle est, au physique, l'incommensurable augmentation de malpropreté, de puanteur et de corruption qui résulte du rapprochement des hommes. $»^{49}$

Que faire alors face à ce mal qui affecte le monde urbain et met en danger ses habitants ? Mercier propose un remède naturel qui offre les prémices d'une médecine environnementale ${ }^{50}:$ «On sait que les végétaux tendent à conserver l'atmosphère dans un état de salubrité, à la purger même de toute corruption; voilà pourquoi les anciens environnaient leurs temples et leurs places publiques de grands arbres : pourquoi ne les imiterions-nous pas? $»^{51}$ Jacques de Horne propose, quant à lui, qu'on favorise « la libre circulation de l'air qu'on y respire, en détruisant tous les obstacles qui peuvent l'intercepter [...] et en éloignant des habitations tous les foyers de malpropreté et de corruption $»^{52}$. Parallèlement à ces projets d'aération citadine, une vaste opération de transfert de déchets d'un espace à un autre s'organise. Comme le souligne Sabine Barles, ce qui distingue la lutte contre la pollution (lutte qui gagnera en intensité au XIX siècle), "c'est la volonté de corrompre là pour assainir ici. Là : hors la ville, en banlieue, sous le sol. Ici : dans la ville. Celle-ci n'est probablement devenue plus saine que parce qu'elle s'est déchargée ailleurs des miasmes délétères qui souillaient son sol $»^{53}$. On expulse donc le miasme, d'un endroit à un autre, sans pour autant l'éradiquer.

Mercier, toutefois, va plus loin en célébrant les vertus de la chimie, science la plus apte à lutter contre ce problème crucial: "On sait aujourd'hui ce qu'on avait si longtemps ignoré, ce qu'est l'air méphitique et de quelle manière on peut combattre ses influences dangereuses et meurtrières. Les bienfaits de la chimie deviennent chaque jour plus nombreux, et donnent des moyens qui intéressent essentiellement l'humanité $\aleph^{54}$. La chimie, qui captive les penseurs de la période, apparaît comme la meilleure arme contre la corruption. Aussi Mercier encourage-t-il les autorités publiques « à interroger souvent la chimie, afin de connaître les moyens que l'art emploie pour détruire ces foyers pestilentiels qui tuent la santé. Une inspection active et surveillante corrigerait le défaut qui résulte d'une vaste population $»^{55}$. Le lexique disciplinaire employé par Mercier est symptomatique des nouveaux impératifs hygiéniques de l'époque. "Au XVIII siècle", rappellent Roger Kempf et Jean-Paul Aron, «la surveillance sanitaire se développe, couvrant, de proche en proche, l'intimité des foyers, la vie collective et la structure du 
milieu ambiant [...]. Servie par la police, sa main-d'œuvre, et la justice, sa caution, l'hygiène travaille à la normalisation $»^{56}$. L'hygiénisme et son principal représentant, l' homo medicus, nouveau garant de la salubrité du monde moderne, visent à assainir la société de ses multiples tares, physiques et psychologiques : "Le médecin est arrivé ", annonce Foucault, médecin prêt à combattre un «mal ayant pris déjà les espèces ambiguës du Fermenté, du Corrompu, des exhalaisons viciées, des chairs décomposées ${ }^{57}$.

\section{De la corruption du sang}

Parmi les foyers de corruption potentiels, le sang, au XVIII ${ }^{e}$ siècle, demeure au centre de toutes les attentions. Car le sang est tout dans l'Ancien Régime : il assure la grandeur ou la décadence d'un individu ; il donne son autorité à la noblesse et fonde son pouvoir ${ }^{58}$. C'est ce même sang qui en promet la mort ou, pire encore, le déclassement. Dans son article "Corruption du sang» de l'Encyclopédie, l'abbé Edme-François Mallet propose la définition juridique suivante : «Les Anglais appellent ainsi la tache imprimée sur tous les descendants d'un criminel de lèse-majesté, qui les rend incapables des charges et emplois publics, et les dégrade de noblesse s'ils sont gentilshommes. ${ }^{59}$ Si la dégradation du sang renvoie au stigmate social et à la tache indélébile transmise aux générations futures, elle l'est aussi au sens physique, le relâchement des mœurs entrainant un appauvrissement du sang : " Au XVIII ${ }^{e}$ siècle, les privilégiés, usés par une suprématie millénaire, s'éteignent dans la luxure; leur cœur, symbole de vaillance, s'effémine, leur sang, vecteur de perfection, s'appauvrit. $\rrbracket^{60}$ Le sang jadis pur et noble, versé héroïquement sur les champs de bataille s'est corrompu avec la vie de salons. D'où l'obsession sanguine qui traverse la littérature comme la médecine des Lumières, et plus particulièrement sa tare qu'est la vérole, petite ou grosse.

Alors que la syphilis, ou grosse vérole, marque le xix ${ }^{e}$ siècle de son empreinte délétère ${ }^{61}$, la petite vérole, ou variole, occupe tous les esprits au $\mathrm{xVIII}^{\mathrm{e}}$ siècle: cette "maladie affreuse et cruelle, dont nous portons le germe dans notre sang, détruit, mutile ou défigure un quart du genre humain $»^{62}$, déplore Charles-Marie de la Condamine dans son Mémoire sur l'inoculation de la petite vérole du 24 avril 1754. Sans distinction de rang, cette maladie issue "d'un sang impur et chargé de miasme putride ${ }^{63}$ exerce sa force corrosive et accélère la corruption du corps. Elle inquiète d'autant plus qu'elle se transmet par contagion de manière insidieuse: "elle se fomente dans nos humeurs, au moyen des crudités ou de la corruption qui s'y trouvent, et ce venin peut se garder longtemps sans se manifester $\aleph^{64}$. Le drame de la petite vérole, dite distincte ou confluente, selon la répartition des pustules sur l'épiderme, est que ses stigmates s'offrent à la vue de tous, sous la forme d'une « picote » qui grêle la peau. C'est en observant ces foules de défigurés, cette population enlaidie dans la fleur de la jeunesse, que Mercier s'indignera avec d'autres de l'absence de traitement adéquat : « Pourquoi le Parisien s'obstine-t-il à voir le nez et les joues de ses filles rongés et cicatrisés, leurs yeux éraillés, lorsqu'elles pourraient conserver ce poli qui, avec la grâce qui les anime, en ferait les plus charmantes créatures de l'Europe ? ${ }^{65}$ Face à la nocivité du mal du siècle, qui coûta la vie à Louis XV et emporta de 50000 à 80000 personnes par an selon les estimations ${ }^{66}$, philosophes et savants réclament le recours à l'inoculation. Cette pratique réparatrice, tant sur le plan esthétique que médical, a été popularisée en France par les médecins suisses SamuelAuguste Tissot (1728-1797), auteur en 1754 de L'Inoculation justifiée, et Théodore Tronchin (1709-1781), auteur en 1765 de l'article «Inoculation» de l'Encyclopédie. Face au corps 
marqué dans sa chair par la terrible maladie, la variolisation préventive annonce «la revanche d'un corps qui peut s'exhiber ${ }^{67}$, symbole d'une pureté retrouvée.

De retour d'Angleterre où la pratique de l'inoculation y est répandue grâce notamment à Lady Mary Wortley Montagu (1690-1762), Voltaire consacre au brûlant sujet la onzième de ses Lettres philosophiques (1734), intitulée "Sur l'insertion de la petite vérole » ${ }^{68}$. Les Anglais, plus avancés dans ce domaine, pratiquent cette «insertion» depuis de nombreuses années, sauvant ainsi leurs enfants d'une mort presque certaine. Considérés par le reste de l'Europe chrétienne comme des criminels pour oser insérer une substance aussi affreuse dans le corps frêle de jeunes individus, les Anglais accusent en retour leurs détracteurs de lâcheté : "Quoi donc?", demande un Voltaire grinçant, « est-ce que les Français n'aiment point la vie? est-ce que leurs femmes ne se soucient point de leur beauté ? ${ }^{69}$ Le débat s'internationalise et revêt un tour idéologique en opposant, d'une part, une nation moderne luttant contre la maladie au moyen d'une pratique nouvelle et efficace, et, d'autre part, une Europe frileuse, peu encline à l'innovation scientifique et prise au piège du discours religieux sur cette maladie.

En quoi consiste l'inoculation, ou «insertion» pour parler la langue de l'époque? Laissons à Voltaire le soin d'en relater la technique :

Les femmes de Circassie sont de temps immémorial dans l'usage de donner la petite vérole à leurs enfants, même à l'âge de six mois, en leur faisant une incision au bras, et en insérant dans cette incision une pustule qu'elles ont soigneusement enlevée du corps d'un autre enfant. Cette pustule fait, dans le bras où elle est insinuée, l'effet du levain dans un morceau de pâte; elle y fermente, et répand dans la masse du sang les qualités dont elle est empreinte. ${ }^{70}$

La description de l'inoculation par Voltaire est intéressante à plus d'un titre. Elle témoigne d'une nouvelle compréhension du pouvoir de la corruption. L'inoculation, " opération par laquelle on communique au corps sain la petite vérole par application, ou par insertion $»^{71}$, n'est autre en effet qu'une corruption provoquée. Il ne s'agit plus d'évacuer la substance virulente, mais d'en insérer une certaine dose afin de permettre à l'organisme de se constituer un rempart contre la future maladie au moyen de ces mêmes impuretés. Delacoste, dans sa Lettre sur l'inoculation de 1723, rappelle à propos de cette méthode que « les Anglais la nomment inoculation, terme de jardinage, qui veut dire greffe $»^{72}$. En effet, « inoculer» vient du latin inoculare, "greffer en écusson», tout comme " insertion", qui vient du latin insitio, "action de greffer». Si l'on se réfère à l'article «Greffe [Jardinage] » de l'Encyclopédie, on peut lire qu'elle est « le triomphe de l'art sur la nature $»^{73}$. L'inoculation, nommée " petite vérole artificielle ", est elle aussi, à sa manière, un triomphe de l'art sur la nature par son aptitude à juguler la petite vérole naturelle. "Par ce moyen ", poursuit l'article, « on force la nature à prendre d'autres arrangements, à suivre d'autres voies, à changer ses formes, et à suppléer le bon, le beau, le grand à la place de l'abject $»^{74}$. Si l'art de la greffe « est ce que l'on a imaginé de plus ingénieux pour la perfection de la partie de l'agriculture qui en fait l'objet $~^{75}$, l'inoculation, en tant que greffe médicale, vise également le perfectionnement du corps humain dans sa lutte contre la corruption.

L'inoculation d'une dose de matière corrompue provoque une fermentation, tel le « levain dans un morceau de pâte ». Comme le précise Diderot, «ce sont les ferments étrangers qui introduisent la corruption, c'est par eux qu'elle commence, continue et s'achève ${ }^{76}$. Cette idée de fermentation est tout aussi centrale que celle de corruption pour les hommes des Lumières. La portée médicale, puis symbolique, de cette découverte est 
immense ; il ne s'agit plus d'extraire le caractère nocif, en affaiblissant l'organisme, mais de l'intégrer, pour en conserver les qualités et s'en servir comme protection future. Ainsi, remarque Catriona Seth, «la technique de l'inoculation marque une révolution de la médecine qui passe de l'ère de la réaction à celle de l'action préventive $»^{77}$. L'inoculation, «la plus belle découverte qui ait été faite en médecine pour la conservation des hommes ${ }^{78}$, déclare Diderot, inaugure une vaste reconsidération de la corruption et de ses qualités inhérentes. Car, face à la corruption, la médecine traditionnelle a toujours procédé par saignée ou par purgation ${ }^{79}$. C'est d'ailleurs la méthode préconisée dans l'article «Vérole, petite » de l'Encyclopédie :

Dans le premier temps, qui est celui de la couve ou de l'ébullition, on doit généralement saigner pour détendre et relâcher la peau, et aider l'éruption; mais on saignera moins que dans une inflammation ordinaire. On ordonnera ensuite un émétique ou un purgatif dans le dessein d'évacuer les premières voies, ou des lavements légèrement purgatifs..$^{80}$

Le vocabulaire est explicite : on saigne, on purge, on lave. La saignée et la purge reposent sur un principe de catharsis, ou purification, qui vise à drainer le mal afin de l'expulser hors de l'organisme ${ }^{81}$. La saignée consiste en une évacuation contrôlée d'une certaine quantité de sang: la veine ou l'artère est incisée à l'aide d'une lancette, de manière à ce que le mal quitte l'organisme avec le sang qui s'écoule. Ceci dit, la pratique n'est pas sans danger. Trop souvent la vie quitte le corps en même temps que la maladie, à moins qu'il ne défaille, au bord de l'épuisement, ce qui le prive de forces pour combattre le mal ${ }^{82}$.

En tant que pratique radicalement nouvelle au XVIII ${ }^{e}$ siècle, l'inoculation variolique pose indubitablement le problème du risque et de la responsabilité, problème que d'Alembert aborde dans ses Réflexions sur l'inoculation lues à l'Académie royale des sciences de Paris en 1760 : « On a trop confondu l'intérêt que l'État en général peut avoir à l'inoculation, avec celui que les particuliers peuvent y trouver [...]. » ${ }^{83}$ Ainsi, «si l'inoculation peut donner la mort, l'État, comme nous l'avons vu, n'est pas en droit d'obliger les citoyens à s'y soumettre. Mais il doit encore moins les en empêcher [...]. ${ }^{84}$ Les questions éthicomédicales soulevées à juste titre par cette nouvelle pratique nécessitent que l'on réfléchisse au problème de l'« expérimentation de masse », comme l'explique Chamayou :

La spécificité de l'inoculation, si on la comprend comme une expérimentation, c'est qu'elle ne se restreint pas à des catégories déterminées de corps vils, mais qu'elle s'étend à l'ensemble de la population, prise comme cible d'une politique sanitaire qui apparaît en même temps comme une expérimentation de masse - les corps vils n'étant plus, dans ce modèle, une fraction avilie de la population mais, en un certain sens, la masse de la population elle-même. ${ }^{85}$

L'inoculation, on le voit, tisse des liens étroits entre savoir et pouvoir, entre une pratique médicale et les moyens de l'administrer. Cette "politique sanitaire ", fruit d'une lutte généralisée contre la corruption, laisse un certain nombre de questions sans réponses: "La Science (et l'État) sont-ils en droit de compter pour cela sur une dissolution de la volonté propre de chacun dans une politique générale censée améliorer le sort de la communauté ? $\gg^{86} \mathrm{Au}$ prix de quels sacrifices peut-on légitimer le combat contre la corruption?

\section{De la corruption et de la génération}

La face de l'univers, si l'on prête attention aux individus, n'est jamais la même pendant deux instants; de quelque côté que l'on regarde, on n'aperçoit que générations et corruptions; encore les corps ne passent-ils d'un de ces termes à 
l'autre, que par des degrés intermédiaires, qui produisent des variétés infinies par les nuances délicates qui s'y font remarquer; il n'y a, en un mot, de repos nulle part, il n'y en a dans aucun temps. ${ }^{87}$ sur les antisceptiques, la génération et la corruption sont à la fois opposées et corrélées pour décrire un univers en mouvement constant. Dans ce rapport de forces, les deux principes agissent à poids égal : «la corruption d'une chose est toujours la génération d'une autre », observe d'Alembert en reprenant la maxime aristotélicienne ${ }^{88}$. De même, celui-ci établit que "génération est opposée à corruption, qui est la destruction d'une chose qui existait », et que pour les anciens philosophes, « la génération d'une chose est proprement la corruption d'une autre ${ }^{89}$. C'est de la sorte que dans la philosophie et la science occidentales, le couple génération-corruption s'est constitué : corruptio unius generatio alterius. Intimement entrelacée avec la génération, dans un jeu de va-et-vient sans fin, la corruption n'est qu'un moment, une promesse, une étape dans le devenir de quelque chose d'autre. C'est en cela, précise d'Alembert, que cette dernière diffère de l'altération : «Une chose est dite altérée lorsqu'elle n'est pas tellement changée qu'on ne la puisse reconnaître, et qu'elle conserve encore son ancien nom : mais après la corruption, ni l'un ni l'autre ne subsistent plus. ${ }^{90}$ La corruption est envisagée ainsi comme un rouage essentiel de la machine complexe qu'est la vie. Elle est avant tout puissance de transformation : "Mais comme dans la génération aucune matière n'est vraiment créée ", poursuit d'Alembert, " ainsi dans la corruption rien n'est réellement anéanti, que cette modification particulière qui constituait la forme d'un être, et qui le déterminait à être de telle ou telle espèce $»^{91}$.

Ce problème précis de la forme d'un être et des différentes modifications qu'il subit au cours de son existence est au cœur de "cette informe et dangereuse production $»^{92} \mathrm{de}$ Diderot qu'est Le Rêve de d'Alembert (1769). Il n'est pas anodin que d'Alembert soit le protagoniste de ce texte, forme elle-même corrompue du dialogue philosophique classique. Voici comment Diderot détaille les grandes lignes du Rêve à Sophie Volland dans sa lettre du 2 septembre 1769 : «Je crois vous avoir dit que j'avais fait un dialogue entre d'Alembert et moi. En le relisant, il m'a pris la fantaisie d'en faire un second, et il a été fait. Les interlocuteurs sont d'Alembert qui rêve, Bordeu et l'amie de d'Alembert, Mlle de l'Espinasse. Il est intitulé Le Rêve de d'Alembert. Il n'est pas possible d'être plus profond

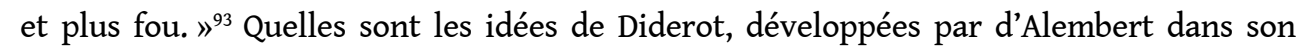
sommeil ? Diderot, nous rappelle Jacques Roger, est le philosophe des Lumières «qui a attribué le plus d'importance aux problèmes biologiques ${ }^{94}$. Parmi cette multitude de problèmes, la question de la génération l'intéresse au plus haut point ${ }^{95}$. Les mécanismes de la génération comme ceux de la corruption restent nimbés de mystère malgré les progrès accomplis. Aussi Diderot est-il conscient d'être arrivé à un moment important de l'histoire des sciences: "Nous sommes à l'aube d'une révolution $»^{96}$, déclare-t-il. Révolution scientifique s'entend, qui a pour ambition d'expliquer comment la matière se fait et se défait.

Le Rêve de d'Alembert est la systématisation du matérialisme diderotien, qui veut que l'homme soit dépendant du biochimisme universel. Comme l'explique Diderot à travers la réflexion onirique de d'Alembert, l'essentiel de la vie repose sur la fermentation générale de la matière. En effet, la matière inerte crée du vivant suite à la fermentation déclenchée par un processus de corruption. Diderot ne mentionne-t-il pas d'ailleurs «le commencement où la matière en fermentation faisait éclore l'univers [...] $\gg^{97}$ ? Il s'inscrit 
en cela dans la lignée de la philosophie épicurienne, et suit le chemin tortueux de la fermentation (processus organique que les personnages du texte évoquent à plusieurs reprises ${ }^{98}$. D'Alembert, rêvant, se demande ce qu'on répondrait aujourd'hui à Épicure lorsque celui-ci «assurait que la terre contenait les germes de tout, et que l'espèce animale était le produit de la fermentation » pour s'exclamer ensuite que «l'éléphant, cette masse énorme, organisée, [serait] le produit subit de la fermentation! Pourquoi non? $\|^{99}$ L'origine du vivant s'en trouve alors éclairée et incite Diderot à intensifier sa réflexion sur la génération en faisant dire à d'Alembert :

Le prodige, c'est la vie, c'est la sensibilité ; et ce prodige n'en est plus un... Lorsque j'ai vu la matière inerte passer à l'état sensible, rien ne doit plus m'étonner... Quelle comparaison d'un petit nombre d'éléments mis en fermentation dans le creux de ma main, et de ce réservoir immense d'éléments divers épars dans les entrailles de la Terre, à sa surface, au sein des mers, dans le vague des airs !... ${ }^{100}$

Cette observation permet à d'Alembert de conclure scientifiquement : «Vous avez deux grands phénomènes, le passage de l'état d'inertie à l'état de sensibilité ; et les générations spontanées. $\aleph^{101}$ Ce passage de la sensibilité inerte à la sensibilité active, qui est au cœur du Rêve de d'Alembert, est donc redevable de la fermentation qui s'opère dans l'ordre naturel ${ }^{102}$.

Le principe de fermentation est indissociable de celui de corruption dans l'explication de l'apparition et du développement de la vie. Comme le souligne Michel Delon, «l'idée de fermentation prolonge celles de corruption et de décomposition. Elle semble solidaire originellement d'une vision de l'histoire comme décadence. $»^{103} \mathrm{En}$ effet, selon l'article «Fermentation [Chimie] » de l'Encyclopédie, « la fin ou l'effet principal et essentiel de la fermentation, c'est la décomposition du corps fermentant, la séparation et l'atténuation de ses principes ${ }^{104}$. La décomposition est l'aboutissement de la fermentation, assurant le changement de forme de l'organisme. Il existe à l'époque un lien étroit entre les différentes terminologies de la rupture et du changement: les penseurs des Lumières, observe Delon, « empruntent à la chimie la notion de fermentation, à la médecine celle de crise, à l'astronomie celle de révolution. Les trois termes désignent une augmentation de l'énergie intérieure qui débouche sur une modification du système $»^{105}$. La modification en question n'est autre que le résultat de l'action conjuguée de la fermentation et de la corruption, laquelle s'opère à l'intérieur de l'organisme.

Diderot déploie une «vision de l'histoire comme décadence » dans la Lettre sur les aveugles lorsqu'il vient à définir ce qu'est le monde : «Un composé sujet à des révolutions, qui toutes indiquent une tendance continuelle à la destruction ; une succession rapide d'êtres qui s'entresuivent, se poussent et disparaissent: une symétrie passagère; un ordre momentané. $»^{106}$ Cette vision déclinante de l'univers apparaît également dans l'article « Homme » qu'il a rédigé pour l'Encyclopédie:

Tout change dans la nature, tout s'altère, tout périt. Lorsque le corps a acquis son étendue en hauteur et en largeur, il augmente en épaisseur ; voilà le premier point de son dépérissement; elle commence au moment où la graisse se forme [...]. Alors les membranes deviennent cartilagineuses, les cartilages osseux, les os plus solides, et les fibres plus dures; la peau se sèche, les rides se forment, les cheveux blanchissent, les dents tombent, le visage se déforme, et le corps s'incline vers la terre à laquelle il doit retourner. ${ }^{107}$

Face à la dégradation inévitable du corps humain, à l'échelle de l'individu comme à l'échelle de l'humanité, le d'Alembert fictif du Rêve se pose les questions suivantes : «Qui sait à quel instant de la succession de ces générations animales nous en sommes? Qui sait 
si ce bipède déformé qui n'a que quatre pieds de hauteur, qu'on appelle encore, dans le voisinage du pôle, un homme, et qui ne tarderait pas à perdre ce nom, en se déformant un peu davantage, n'est pas l'image d'une espèce qui passe ? ${ }^{108}$ La déformation de l'espèce inscrit la corruption dans une logique de la dégénérescence ${ }^{109}$.

Pourtant, cette appréciation d'un devenir de l'homme marqué par ses déformations successives au point de se détruire n'enferme pas Diderot dans une pensée strictement négative, comme en témoigne l'article « Naître » qu'il rédige pour l'Encyclopédie:

À proprement parler, on ne naît point, on ne meurt point; on était dès le commencement des choses, et on sera jusqu'à leur consommation. Un point qui vivait s'est accru, développé, jusqu'à un certain terme, par la juxtaposition successive d'une infinité de molécules. Passé ce terme, il décroît, et se résout en molécules séparées qui vont se répandre dans la masse générale et commune. ${ }^{110}$

Non plus naitre et mourir donc, avec leur signification de commencement et de fin, mais croître et décroître, dans l'insistance d'une continuité jamais interrompue: "Tout change. Tout passe. Il n'y a que le tout qui reste. Le monde commence et finit sans cesse. Il est à chaque instant à son commencement et à sa fin $»^{111}$, nous rappelle d'Alembert endormi. Ce qui intéresse Diderot dans le phénomène complexe de la vie, ce n'est pas tant son apparition ou sa disparition que sa capacité à se modifier, à se transformer, en suivant une voie qui lui est propre. En cela, l'homme, comme tout être vivant, est un état changeant, un état provisoire sans commencement ni fin véritable :

La vie est une qualité essentielle et primitive de l'être vivant ; il ne l'acquiert point ; il ne la perd point. Il faut distinguer une vie inerte et une vie active: elles sont entre elles comme la force vive et la force morte : ôtez l'obstacle, et la force morte deviendra force vive : ottez l'obstacle, et la vie inerte deviendra vie active. [...] Les termes de vie et de mort n'ont rien d'absolu; ils ne désignent que les états successifs d'un même être [.... . ${ }^{112}$

Cette redéfinition fondamentale des termes mêmes de "vie» et de "mort» est à rapprocher de la définition proposée par d'Alembert dans le Rêve :

Et la vie ?... La vie, une suite d'actions et de réactions... Vivant, j'agis et je réagis en masse... mort, j'agis et je réagis en molécules... Je ne meurs donc point ?... Non, sans doute, je ne meurs point en ce sens, ni moi, ni quoi que ce soit... Naître, vivre et passer, c'est changer de formes... Et qu'importe une forme ou une autre ? ${ }^{113}$

"Changer de formes », n'est-ce pas là le principe même de la corruption, qui est « l'état par lequel une chose cesse d'être ce qu'elle était $~^{114}$, pour justement en devenir une autre? Cette "suite d'actions et de réactions ", qui entraîne une modification définitive de l'entité d'origine, n'est-elle pas la traduction de cette succession de génération (genesis) et de corruption (phthora), qui imite la translation (phora), comprise ici comme force ou mouvement, selon la physique d'Aristote ? ${ }^{115}$ Dans une lettre du 15 octobre 1759, Diderot donnait à Sophie Volland l'explication suivante : «La seule différence que je connaisse entre la mort et la vie, c'est qu'à présent vous vivez en masse, et que dissous, épars en molécules, dans vingt ans d'ici vous vivrez en détail. »116 Propos qui permettent à Starobinski, d'interpréter le projet encyclopédique de Diderot en ces termes : «Diderot imagine des vicissitudes catastrophiques, une fécondité inépuisable, et de nouvelles formes de vie faisant suite à de grandes destructions. Sa certitude de l'éternité matérielle lui permet d'envisager tous les désastres. Le monde leur survivra, sans que ses molécules perdent leur possibilité de recombinaison. $»^{117}$

39 Aussi Le Rêve de d'Alembert fait-il la part belle au transformisme de l'espèce humaine, et ses protagonistes se laissent séduire à la fin de l'ouvrage par une tératologie sous forme 
de "chèvre-pied" et autre monstruosité, "où la matière cherche toutes les voies possibles pour produire de nouveaux mélanges, de nouvelles formes vivantes $»^{118}$. En effet, une fois posée la nature même de la vie - «la vie est de l'agrégat $»^{119}-$, l'entretien de Bordeu et de Mlle de l'Espinasse se dirige progressivement vers les états limites de cette même vie, autrement dit vers l'anomalie ${ }^{120}$. Diderot dresse alors, à l'intérieur de ses considérations sur la vie, une science des monstres qui poursuit sa réflexion sur les effets dévastateurs de la corruption. La discussion se poursuit entre Bordeu et Mlle de l'Espinasse, qui finissent par aborder la question délicate du « mélange des espèces » ainsi que de l'hybridation. Ce qui est l'occasion pour le docteur Bordeu, dans la partie intitulée Suite de l'entretien précédent, de développer ses théories sur "l'art de créer des êtres » ${ }^{121}$, tout en abordant le sujet sous ses différentes facettes, qu'elles soient d'ordre physique, moral ou poétique. Mlle de l'Espinasse en vient à s'interroger sur la provenance de tous " ces goûts abominables» qui constituent le propos final de la discussion, alors que les "chèvre-pieds", qualifiés d'« effrénés dissolus", refont leur apparition. Bordeu s'empresse alors de répondre, non sans une pointe d'ironie, qu'ils sont le résultat « d'une pauvreté d'organisation dans les jeunes gens, et de la corruption dans la tête des vieillards $»^{122}$. Pauvreté d'organisation d'une part, corruption mentale d'autre part, le corps et l'esprit étant tous deux soumis à la loi de la dégénération. On ne saurait mieux conclure un entretien comme Le Rêve de d'Alembert où la question de la génération et de la corruption aura été le grand sujet, toujours discuté, jamais épuisé.

Par sa propension intrinsèque à générer de nouvelles formes, la corruption participe activement à la révolution qui s'opère au XVIII ${ }^{e}$ siècle : révolution politique bien sûr, qui mobilise de nouveaux pouvoirs en libérant dans l'espace public des énergies jusqu'alors inédites, mais aussi révolution scientifique liée à l'émergence de nouveaux savoirs qui façonneront les siècles à venir ${ }^{123}$. Si, comme le rappelle l'article «Putréfaction, Putréfier [Chimie]», «les substances corrompues donnent la meilleure terre pour fertiliser les champs $»^{124}$, la corruption à l'âge des Lumières peut être qualifiée métaphoriquement de fertilisant hors pair pour les champs nouvellement défrichés de la connaissance. Sade, sensible à la question, en prolonge ainsi l'idée dans son roman philosophique Aline et Valcour : « Lorsque le grain germe dans la terre, lorsqu'il se fertilise et se reproduit, est-ce autrement que par corruption, et la corruption n'est-elle pas la première des lois génératrices? ${ }^{125}$ Simple interrogation qui rappelle que le propre de la corruption n'est autre que de donner vie à un nouvel organisme, à une nouvelle société, à un nouveau savoir.

\section{NOTES}

1. «Dernière réponse de J.-J. Rousseau de Genève [à Bordes] », in Jean-Jacques Rousseau, CEuvres complètes, t. 3, Paris, Gallimard, coll. « Bibliothèque de la Pléiade », 1964, p. 73.

2. Comme nous l'avons établi dans un précédent travail, les Lumières regroupent «l'ensemble des phénomènes intellectuels convoqués par une société émancipée afin de résoudre le problème majeur de la corruption » (Ronan Chalmin, Lumières et corruption, Paris, Champion, 2010, p. 10). Le 
présent article est une continuation de notre réflexion sur le rôle propédeutique joué par la corruption au XVIII ${ }^{\mathrm{e}}$ siècle, cette fois dans le domaine des sciences.

3. Charles Mauriac, article "Onanisme", Dictionnaire de médecine et de chirurgie pratiques, t. 24, Paris, Baillière, 1877, p. 496.

4. Voir en particulier Mona Ozouf, «La Révolution française et la formation de l'homme nouveau ", in L'Homme régénéré. Essais sur la Révolution française, Paris, Gallimard, 1989, p. 116-157 ; Robert Favre, « La Révolution : mort et régénération, ou la France "phénix" ? ", Dix-huitième siècle, $n^{\circ}$ 23, 1991, p. 331-344 ; Antoine de Baecque, «La régénération, corps merveilleux ou corps dressé du nouvel homme révolutionnaire", in Le Corps de l'histoire. Métaphores et politique (1770-1800), Paris, Calmann-Lévy, 1993, p. 165-194 ; Xavier Martin, Régénérer l'espèce humaine. Utopie médicale et Lumières (1750-1850), Bouère, DMM, 2008.

5. Jacques Guillerme, «Le malsain et l'économie de la nature », Dix-huitième siècle, $\mathrm{n}^{\circ}$ 9, 1977, p. 63.

6. Jean le Rond d'Alembert, "Corruption", in Denis Diderot et Jean Le Rond d'Alembert, Encyclopédie ou dictionnaire raisonné des sciences, des arts et des métiers (1751-1772), t. 4, p. 278 (dorénavant abrégé en Encycl. suivi du tome et de la page). Toutes les citations utilisées dans cet article sont extraites du site The ARTFL Encyclopédie, consultable à l'adresse électronique suivante : http://encyclopedie.uchicago.edu/.

7. Alain Rey donne de ce mot, emprunté au latin corruptio, la définition suivante: «La philosophie s'est servie de corruption pour rendre le concept grec de phthora (opposé à celui de genesis), désignant l'événement par lequel une chose cesse d'être telle qu'on puisse encore la désigner par le même nom » (Article "Corruption», Dictionnaire historique de la langue française, sous la dir. d'Alain Rey, Paris, Le Robert, 2016, p. 568).

8. Michel Serres, "L'Antéchrist: une chimie des sensations et des idées ", in Hermès IV. La distribution, Paris, Minuit, 1977, p. 178.

9. Voir, entre autres, Joachim Faiguet de Villeneuve, L'Économe politique. Projet pour enrichir et pour perfectionner l'espèce humaine, Paris, chez Moreau, Pissot, Knapen, Brocas et Humblot, 1763; Charles Auguste Vandermonde, Essai sur la manière de perfectionner l'espèce humaine, 2 vol. , Paris, Vincent, 1756 ; Jean-Jacques Millot, L'Art d'améliorer et de perfectionner les hommes, au moral comme au physique, 2 vol., Paris, impr. de Migneret, 1801 ; Benjamin Constant, De la perfectibilité de l'espèce humaine, in Mélanges de littérature et de politique, Paris, Pichon et Didier, 1829, p. 387-415.

10. Grégoire Chamayou, Les Corps vils. Expérimenter sur les êtres humains aux XVIII et XIX ${ }^{e}$ siècles, Paris, La Découverte, 2008, p. 385.

11. C'est ainsi que le chimiste français Gabriel François Venel présente G.-E. Stahl dans l'article «Chymie » de l'Encyclopédie tout en qualifiant le style de Stahl de « dur, serré, embarrassé, et plus barbare en latin que la qualité d'écrivain moderne ne le comporte» (Encycl. 3, p. 436).

12. Voir Bernadette Bensaude-Vincent, «Phlogistique», in Michel Delon (dir.), Dictionnaire européen des Lumières, Paris, PUF, coll. « Quadrige », 2007, p. 984-986.

13. Georg-Ernst Stahl, De la nécessité d'éloigner de la doctrine médicale tout ce qui lui est étranger [1706], in CEuvres médico-philosophiques et pratiques (traduites et commentées par T. Blondin), t. 2, Paris, Baillière, 1859-1864, p. 224.

14. Stahl, Véritable distinction à établir entre le mixte et le vivant du corps humain [1707], op. cit, t. 2, p. 369.

15. André Pichot, Histoire de la notion de vie, Paris, Gallimard, 1993, p. 461.

16. Stahl, op. cit., t. 2, p. 227.

17. Stahl, Vraie théorie médicale [1708], op. cit., t. 3, p. 42.

18. Encycl. 3, p. 413.

19. Stahl, ibid., p. 43.

20. Xavier Bichat, Recherches physiologiques sur la vie et la mort [1800], Genève-Paris-Bruxelles, Alliance culturelle du livre, 1962, p. 43. 
21. Stahl, op. cit., t. 3, p. 43.

22. «La nature est le médecin des maladies. La nature trouve pour elle-même les voies et moyens, non par intelligence. [...] La nature, sans instruction et sans savoir, fait ce qu'il convient » Hippocrate, "Épidémies ", $6^{\mathrm{e}}$ livre, $5^{\mathrm{e}}$ section, in CEuvres complètes, t. 5, Paris, Baillière, 1839-1861, p. 315.

23. Voir Paul Hoffmann, «L'âme et les passions dans la philosophie médicale de Georg-Ernst Stahl », Dix-huitième siècle, $\mathrm{n}^{\circ}$ 23, 1991, p. 31-43.

24. Stahl, De la nécessité d'éloigner de la doctrine médicale tout ce qui lui est étranger, op. cit., t. 2, p. 255.

25. Stahl, ibid., p. 249.

26. Stahl y consacre sa dissertation inaugurale, Des intestins, de l'art de bien connaitre et de guérir leurs affections morbides et leurs symptômes, donnée publiquement à Iéna le 20 janvier 1684 .

27. Stahl, Distinction du mixte et du vivant [1707], op. cit., t. 2, p. 426-427.

28. Alain Corbin, Le Miasme et la jonquille. L'odorat et l'imaginaire social, XVIII ${ }^{e}$-XIX ${ }^{e}$ siècles, Paris, Champs/Flammarion, 1986, p. 17.

29. Richard Etlin, «L'air dans l'urbanisme des Lumières ", Dix-huitième siècle, nº 9, 1977, p. 123.

30. Hippocrate, Traité des airs, des eaux et des lieux (nouvelle traduction d'A. Coray en 1800 en 2 volumes). Sur l'air et ses différentes problématiques, voir Cyrille Simonnet, Brève histoire de l'air, Versailles, Éditions Quæ, 2014.

31. Corbin, op. cit., p. 17.

32. Voir, par exemple, Guillaume-Lambert Godart, "Septicologie ou dissertation sur les antiseptiques", in Dissertations sur les antiseptiques qui ont concouru pour le prix proposé par l'Académie des sciences, arts et belles-lettres de Dijon en 1767, Dijon, chez François des Ventes, Paris, chez des Ventes de la Doué, 1769.

33. Anon., «Putréfaction, Putréfier [Chimie] », Encycl. 13, p. 587.

34. Georges Vigarello, Histoire des pratiques de santé. Le sain et le malsain depuis le Moyen Âge, Paris, Le Seuil, 1999, p. 178.

35. Roy Porter et Georges Vigarello, "Corps, santé et maladies », in Alain Corbin, Jean-Jacques Courtine, Georges Vigarello (dir.), Histoire du corps, t. 1, Paris, Le Seuil, 2005, p. 369.

36. Sabine Barles, « Les villes transformées par la santé, $\mathrm{XVIII}^{\mathrm{e}}-\mathrm{XX}^{\mathrm{e}}$ siècles », Les Tribunes de la santé, $n^{\circ} 33,2011$, p. 32.

37. Alexandre Tournon, Moyens de rendre parfaitement propres les rues de Paris, et celles des autres villes du royaume, Paris, chez Lescarpart, 1789, p. 3.

38. Louis Sébastien Mercier, "Noyés. Vapeurs de charbon », Tableau de Paris, in Paris le jour, Paris la nuit, Paris, Robert Laffont, 1990, p. 54.

39. Mercier, «L'air vicié », op. cit., p. 49.

40. Ibid., p. 48.

41. Mercier, « Amélioration », op. cit., p. 116.

42. Mercier, «L'air vicié », op. cit., p. 48.

43. Corbin, op. cit., p. 57.

44. Mercier, « Bicêtre », op. cit., p. 243.

45. Michel Foucault, Histoire de la folie à l'âge classique, Paris, Gallimard, 1972, p. 376.

46. Louis-Michel Musquinet de la Pagne, Bicêtre réformé. Établissement d'une maison de discipline, Paris, chez Garnéri, 1789, p. 18.

47. Foucault, op. cit., p. 376.

48. Voir Rudy Le Menthéour, qui examine l'hygiène physico-morale de Rousseau (et notamment ses idées sur l'air) dans La Manufacture de maladies. La dissidence hygiénique de Jean-Jacques Rousseau (Paris, Classiques Garnier, 2011).

49. Michel Thouret, Rapport sur la voirie de Montfaucon, lu le 11 novembre 1788 à la société royale de médecine, Paris, Impr. des Annales d'agriculture, (s. d.), p. 28. 
50. Cette médecine moderne, explique Janković, se fonde sur «la différenciation faite au XVIII siècle entre les qualités médicales des espaces intérieurs et extérieurs" ("the eighteenthcentury differenciation between the medical qualities of indoors and outdoors») (Vladimir Janković, Confronting the Climate. British Airs and the Making of Environmental Medicine, New York, Palgrave Macmillan, 2010, p. 1-2).

51. Mercier, « L'air vicié », op. cit., p. 49.

52. Jacques de Horne, Mémoire sur quelques objets qui intéressent plus particulièrement la salubrité de la ville de Paris, Paris, Impr. J.C. Desaint, 1788, p. 1.

53. Sabine Barles, La Ville délétère. Médecins et ingénieurs dans l'espace urbain, XVIII-XIX ${ }^{e}$ siècles, Paris, Champ Vallon, 1999, p. 324. En conséquence, « l'aval corrompu d'une ville peut devenir l'amont corrompu d'une autre ville» (Sabine Barles, art. cité, p. 36).

54. Mercier, «L'air vicié », Tableau de Paris, p. 50-51.

55. Mercier, « Amélioration », ibid., p. 116.

56. Jean-Paul Aron et Roger Kempf, La Bourgeoisie, le sexe et l'honneur, Bruxelles, Éditions Complexe, 1984, p. 12.

57. Foucault, op. cit., p. 378.

58. Voir André Devyver, Le Sang épuré. Les préjugés de race chez les gentilshommes français de l'Ancien Régime (1560-1720), Bruxelles, Éditions de l’Université de Bruxelles, 1973.

59. Encycl. 4, p. 278-279.

60. Aron et Kempf, op. cit., p. 270.

61. Voir Patrick Wald Lasowski, Syphilis. Essai sur la littérature du XIX ${ }^{e}$ siècle, Paris, Gallimard, 1982 ; voir aussi Jean-Louis Cabanès, «Invention(s) de la syphilis », Romantisme, n 94, 1996, p. 89-109.

62. Charles-Marie de la Condamine, Mémoires pour servir à l'histoire de l'inoculation de la petite vérole. Lus à l'Académie royale des sciences en 1754, 1758 et 1765, Paris, de l'Imprimerie royale, 1768, p. 1.

63. Anon., « Vérole, petite [Médecine] », Encycl. 17, p. 81.

64. Ibid.

65. Mercier, «Inoculation », op. cit., p. 171.

66. Pierre Darmon, La Variole, les nobles et les princes. La petite vérole mortelle de Louis XV, Bruxelles, Éditions Complexe, 1989, p. 32.

67. Catriona Seth, Les Rois aussi en mouraient. Les Lumières en luttent contre la petite vérole, Paris, Desjonquères, 2008, p. 5.

68. Voltaire offre dans l'édition de 1775 la précision suivante : «Cela fut écrit en 1727. Aussi l'auteur fut le premier en France qui parla de l'insertion de la petite vérole ou variole, comme il fut le premier qui écrivit sur la gravitation» (lettre XI [note a]), Lettres philosophiques (présentation par Gerhardt Stenger), Paris, Flammarion, 2006, p. 118).

69. Ibid., p. 121.

70. Ibid., p. 118-119.

71. Tronchin, «Inoculation », Encycl. 8, p. 769.

72. Jean Delacoste, Lettre sur l'inoculation de la petite vérole, comme elle se pratique en Turquie et en Angleterre, adressée à M. Dodart, conseiller d'État et premier médecin du roi, Paris, chez Claude Labottière, 1723, p. 5.

73. Encycl. 7, p. 921.

74. Ibid.

75. Ibid., p. 922.

76. Diderot, « Théosophes », Encycl. 16, p. 258.

77. Catriona Seth, «L'inoculation contre la variole : un révélateur des liens sociaux », Dix-huitième siècle, $\mathrm{n}^{\circ}$ 41, 2009, p. 152.

78. Diderot, « Insertion de la petite vérole », Encycl. 8, p. 788. 
79. «La saignée a été, avec la purgation, la pratique la plus connue et la plus courante de l'ancienne médecine», observe Jacques Léonard ("À propos de l'histoire de la saignée (1600-1900)», Mentalités. Histoire des cultures et des sociétés, ํㅡㄴ 1, 1988, p. 73).

80. Encycl. 17, p. 81.

81. L'article souligne à ce propos que «l'on doit employer les purgatifs, les apéritifs, les fondants mercuriaux, les bains, les sudorifiques, les eaux minérales, le lait... » (Ibid.)

82. «On voit des malades périr après la saignée », conclut l'article « Vérole, petite », en signalant que « Alsaharavius prescrit la saignée jusqu'à la défaillance et jusqu'à l'évanouissement » (Ibid.).

83. D'Alembert, «Réflexions sur l'inoculation », in Euvres de d'Alembert, t. 1, Paris, Bossange, 1821, p 480-493.

84. Ibid.

85. Chamayou, op. cit., p. 116.

86. Seth, art. cité, p. 153.

87. Godart, op. cit., p. 283-284.

88. D'Alembert, «Corruption », Encycl. 4, p. 278. Aristote donne cette définition classique : «La génération d'une chose est toujours la corruption d'une autre, et la corruption d'une chose, la génération d'une autre.» (Aristote, De la génération et de la corruption, I, 3, Paris, Librairie philosophique J. Vrin, 1998, p. 35.) Dans son article «Impérissable », Diderot reprend la même idée : « La destruction d'une chose a été, est et sera à jamais la génération d'une autre » (Encycl. 8, p. 593).

89. D’Alembert, « Génération [Physique] », Encycl. 7, p. 558.

90. Ibid.

91. D’Alembert, « Corruption », Encycl. 4, p. 278.

92. Diderot, Correspondance, t. 9, Paris, Minuit, 1955-1970, p. 158.

93. Diderot, Lettres à Sophie Volland, éd. A. Babelon, t. 2, Paris, Gallimard, 1950, p. 224. Sur l'originalité du dialogue écrit par Diderot, voir Colas Duflo, «Et pourquoi des dialogues en des temps de systèmes?» (Diderot Studies, $\mathrm{n}^{\circ} 28,2000$, p. 95-109).

94. Jacques Roger, Les Sciences de la vie dans la pensée française du XVIII siècle. La génération des animaux de Descartes à l'Encyclopédie, Paris, Armand Colin, 1963, p. 585.

95. Sur l'importance du mot "génération » chez Diderot, voir Annie Ibrahim, Le Vocabulaire de Diderot, Paris, Ellipses, 2016, p. 29-30.

96. Diderot, De l'Interprétation de la Nature, in CEuvres philosophiques, Paris, Classiques Garnier, 1998, p. 180.

97. Diderot, Lettre sur les aveugles à l'usage de ceux qui voient, in op. cit., p. 123.

98. Sur la place centrale occupée par la fermentation dans la pensée de Diderot, voir Jean Starobinski, Action et réaction. Vie et aventures d'un couple, Paris, Éditions du Seuil, 1999, p. 70 sq. Voir aussi Fumie Kawamura, Diderot et la chimie. Science, pensée et écriture, Paris, Classiques Garnier, 2013, p. 225 sq.

99. Diderot, Le Rêve de d'Alembert (présentation Colas Duflo), Paris, Flammarion, 2002, p. 95-96.

100. Ibid., p. 96.

101. Ibid., p. 97.

102. Sur la question de la sensibilité au XVIII ${ }^{\mathrm{e}}$ siècle, voir Anne C. Vila, Enlightenment and Pathology: Sensibility in the Literature and Medicine of Eighteenth-Century France, Baltimore, Johns Hopkins University Press, 1998.

103. Michel Delon, L'Idée d'énergie au tournant des Lumières (1770-1820), Paris, PUF, 1988, p. 223.

104. Venel, Encycl. 6, p. 518.

105. Ibid. Rappelons pour information que c'est le docteur Bordeu lui-même qui rédige l'article «Crise [Médecine] » de l'Encyclopédie.

106. Diderot, Lettre sur les aveugles, op. cit., p. 123. 
107. Diderot, « Homme », Encycl. 8, p. 260.

108. Diderot, Le Rêve de d'Alembert, op. cit., p. 95-96.

109. Sur la question de la dégénérescence à cette époque, voir l'étude de Claude-Olivier Doron, L'Homme altéré. Races et dégénérescence (XVII ${ }^{e}$ XIX ${ }^{e}$ siècles), Paris, Champ Vallon, 2016.

110. Diderot, « Naître », Encycl. 11, p. 10.

111. Diderot, Le Rêve de d'Alembert, op. cit., p. 94.

112. Diderot, « Naître ", Encycl. 11, p. 10.

113. Le Rêve de d'Alembert, op. cit., p. 104.

114. D’Alembert, «Corruption », Encycl. 4, p. 278.

115. Voir ici les développements de Starobinski, Action et réaction, op. cit., p. 19-20.

116. Diderot, Lettres à Sophie Volland, op. cit., t. 1, p. 70.

117. Starobinski, Action et réaction, op. cit., p. 301.

118. Jean Starobinski, «Le philosophe, le géomètre, l'hybride ", in Diderot, un diable de ramage, Paris, Gallimard, 2012, p. 264.

119. Diderot, Le Rêve de d'Alembert, op. cit., p. 163.

120. Voir Annie Ibrahim, «Le statut des anomalies dans la philosophie de Diderot », Dix-huitième siècle, n 15, 1983, p. 311-327.

121. Diderot, Le Rêve de d'Alembert, op. cit., p. 171.

122. Ibid., p. 183.

123. Sur l'ensemble de ces questions, voir Michel Serres, «Paris 1800 », in Michel Serres (dir.), Éléments d'histoire des sciences, Paris, Bordas, 1989, p. 503-539.

124. «Putréfaction, Putréfier [Chimie] », Encycl. 13, p. 587.

125. D.A.F de Sade, lettre XXXV, Aline et Valcour, ou le roman philosophique, in Euvres, t. 1, Paris, Gallimard, coll. « Bibliothèque de la Pléiade », 1990, p. 557.

\section{RÉSUMÉS}

La vaste réflexion sur la corruption menée au XVIII ${ }^{\mathrm{e}}$ siècle tant par la science que la philosophie et la littérature a permis une meilleure compréhension des phénomènes du vivant et, en conséquence, une amélioration générale des conditions de vie. Les nouveaux savoirs de la corruption ont identifié celle-ci comme menace tout en reconnaissant son rôle primordial dans le cycle vital. Ils y voient un instrument de progrès, notamment avec les pratiques modernes de l'aération et de l'inoculation. La corruption et sa connaissance sont également au cœur des réflexions sur les mécanismes de la génération et, dans le cas de Diderot, ont servi de source d'inspiration poétique aussi bien que philosophique.

The enormous amount of theorizing on corruption that was carried out in eighteenth-century science, philosophy, and literature enabled a better understanding of the phenomena of the living body and, consequently, an improvement in living conditions. Although they identified corruption as a threat, the works devoted to it recognized corruption's role in vital processes. They also view corruption as an instrument of progress, notably through practices such as aeration and inoculation. Knowledge of corruption also underpinned reflections on the mechanisms of generation - and, in the case of Diderot, served as a source of poetic as well as philosophical inspiration. 
INDEX

Keywords : corruption, Diderot (Denis), d'Alembert (Jean Le Rond), generation, inoculation, Mercier (Louis Sébastien), Stahl (Georg-Ernst)

Mots-clés : corruption, Diderot (Denis), d'Alembert (Jean Le Rond), génération, inoculation, Mercier (Louis Sébastien), Stahl (Georg-Ernst)

\section{AUTEUR}

RONAN CHALMIN

Connecticut College 